\title{
Long non-coding RNA MIR4713HG aggravates malignant behaviors in oral tongue squamous cell carcinoma via binding with microRNA let-7c-5p
}

\author{
BO JIA ${ }^{1,2^{*}}$, XIANGHUAI ZHENG ${ }^{2 *}$, XIAOLING QIU ${ }^{2}$, XIAO JIANG ${ }^{1,2}$, JINGPENG LIU $^{2}$, \\ ZHIJIE HUANG ${ }^{2}$, SHIJIAN XIANG ${ }^{3}$, GUODONG CHEN $^{1 *}$ and JIANJIANG ZHAO ${ }^{2}$
}

\author{
${ }^{1}$ Department of Stomatology, Shunde Hospital, Southern Medical University, Foshan, Guangdong 528308; \\ ${ }^{2}$ Department of Oral Surgery, Stomatological Hospital, Southern Medical University, Guangzhou, Guangdong 510280; \\ ${ }^{3}$ Department of Pharmacy, Seventh Affiliated Hospital of Sun Yat-sen University, Shenzhen, Guangdong 518107, P.R. China
}

Received July 24, 2020; Accepted November 16, 2020

DOI: $10.3892 /$ ijmm.2021.4917

\begin{abstract}
Oral tongue squamous cell carcinoma (OTSCC) is one of the most aggressive pathological types of head and neck squamous cell carcinoma, and presents with rapid local invasion and metastasis. The present study confirmed that the long non-coding (lnc) RNA MIR4713HG was markedly upregulated in both OTSCC tissues and cell lines and associated with poor survival. The present study performed a series of experiments to investigate the impact of MIR4713HG on OTSCC and revealed that upregulation of MIR4713HG had a crucial role in promoting cell proliferation and metastasis of OTSCC cell lines both in vitro and in vivo. By applying bioinformatics analyses, micro RNA let-7c-5p was observed to physically bind with MIR4713HG, and the knockdown of let-7c-5p could counteract the influence of MIR4713HG on OTSCC. Furthermore, the present study demonstrated that let-7c-5p performed its regulating role in OTSCC via affecting the expression level of transmembrane channel like 7 (TMC7). In conclusion, the present study demonstrated that lncRNA MIR4713HG acted as a pro-tumor factor facilitating cell proliferation and metastasis of OTSCC via affecting the let-7c-5p/TMC7 signaling pathway, which presents as a promising therapeutic target in OTSCC.
\end{abstract}

Correspondence to: Professor Guodong Chen, Department of Stomatology, Shunde Hospital, Southern Medical University, 1 Jiazi Road, Lunjiao, Shunde, Foshan, Guangdong 528308, P.R. China

E-mail: chenzejia99@163.com

Professor Jianjiang Zhao, Department of Oral Surgery, Stomatological Hospital, Southern Medical University, 366 South Jiang Nan Road, Haizhu, Guangzhou, Guangdong 510280, P.R. China

E-mail: jianjiangzhao123@sina.com

*Contributed equally

Key words: OTSCC, lncRNA MIR4713HG, let-7c-5p, TMC7, metastasis

\section{Introduction}

Oral tongue squamous cell carcinoma, the sixth most frequent cancer worldwide, is one of the most aggressive pathological types of head and neck squamous cell carcinoma $(1,2)$. OTSCC is a highly malignant cancer that presents with rapid local invasion and distal metastasis and is frequently diagnosed in young individuals, particularly in males $(3,4)$. Although the therapeutic regimens for OTSCC have been greatly improved over the years, clinicians have not observed evident improvement of the 5-year survival rate in patients with OTSCC due to the high recurrence rates $(5,6)$. Generally, OTSCC is a complex disease with extensive genetic/epigenetic alterations (7). Therefore, it is urgent to investigate its potential carcinogens with the hope of identifying new therapeutic targets.

Long non-coding RNAs refers to a group of non-coding RNAs with a length of $>200$ nucleotides (8). When first discovered, IncRNAs were regarded as transcriptional 'noise' generated during the transcription process and held no biological functions $(9,10)$. However, in recent years, more studies have reported that IncRNAs play an important role in a wide range of cellular processes, including cell differentiation, cell apoptosis, cell proliferation, gene transcription, epigenetic modification as well as others (11-13). Furthermore, IncRNAs have been demonstrated to act as crucial regulators in various malignancies (14-16). HOTTIP, one IncRNA in OTSCC, has been reported to suppress the proliferation, migration and invasion of OTSCC by regulating the HMGA2-mediated Wnt/b-Catenin pathway $(17,18)$. Therefore, it is essential to identify the key IncRNAs involved in OTSCC progression in order to help understand the mechanisms underlying this disease.

The present study investigated the expression levels of MIR4713HG in OSTCC tissues and cell lines, and evaluated its influence on cell proliferation, invasion, migration and tumorigenesis of OTSCC. Furthermore, the interaction between MIR4713HG and let-7c-5p, and their downstream target genes were investigated. The findings may help elucidate the molecular mechanisms underlying the carcinogenic process of OTSCC and provide potential therapeutic targets. 


\section{Materials and methods}

RNA sequence data analysis. MIR4713HG and let-7c-5p RNASeq data from OTSCC samples were downloaded from the Cancer Genome Atlas (TCGA) database (https://cancergenome.nih.gov/). All the data are publicly available. MIR4713HG and let-7c-5p were identified according to the Ensembl database (http://www.ensembl.org/index.html, version 89) The edgeR package (19) was used to normalize gene expression of MIR4713HG and let-7c-5p in TSCC and normal tissues.

Cell culture and patient samples. The human OTSCC cell lines (CAL-27, SCC-9, SCC-4, SCC-15 and SCC-25) were purchased from Shanghai Cell Bank of the Chinese Academy of Sciences and the human periodontal ligament fibroblast (HPLF) cell line was purchased from ScienCell Research Laboratories, Inc. (cat. no. 2630). Cells were cultured at $37^{\circ} \mathrm{C}$ in an atmosphere containing $5 \% \mathrm{CO}_{2}$ in RPMI- 1640 medium supplemented with $10 \%$ FBS and $1 \%$ penicillin-streptomycin solution (all from Gibco; Thermo Fisher Scientific, Inc.).

With approval from the institutional review board of the Joint Ethics Committee of the Southern Medical University Health Authority, freshly resected OTSCC tissues (20 pairs) and paraffin-embedded OTSCC sections were obtained from the archives of the Department of Oral Surgery, Stomatological Hospital, Southern Medical University (Guangzhou, China). Each participant provided written informed consent.

RNA interference assay. The short hairpin (sh) RNA targeting MIR4713HG and small interfering (si)RNA targeting transmembrane channel like 7 (TMC7) were synthesized by Shanghai GenePharma Co., Ltd. Sequences of shRNA or siRNAs against specific targets are listed in Table SI. The sequence with the best interfering effect was selected and used in subsequent experiments.

Cell transfection. CAL-27 and SCC-9 cells were transfected with $1 \mu \mathrm{g}$ pcDNA3.1-MIR4713HG and $1 \mu \mathrm{g}$ control pcDNA3.1 plasmids (control-plasmids; both from Shanghai GenePharma Co., Ltd.); $1 \mu \mathrm{g}$ shRNA (sh-MIR4713HG), $1 \mu \mathrm{g}$ negative control shRNA (sh-NC), $100 \mathrm{nM}$ let-7c-5p inhibitors (miR20000064-1-5), and $100 \mathrm{nM}$ let-7c-5p inhibitor controls (miR2N0000001-1-5); $100 \mathrm{nM}$ let-7c-5p-mimics (miR10000064-1-5) and $100 \mathrm{nM}$ let-7c-5p mimic negative controls (miR1N0000001-1-5; all from Guangzhou RiboBio Co., Ltd.) for $48 \mathrm{~h}$ at $37^{\circ} \mathrm{C}$ using Lipofectamine ${ }^{\circledR} 2000$ reagent (Invitrogen; Thermo Fisher Scientific, Inc.), according to the manufacturer's protocol. Transfection efficiency was determined $48 \mathrm{~h}$ post-transfection via reverse transcription-quantitative (RT-q) PCR. Sequences of let-7c-5p inhibitor or let-7c-5p mimics against specific targets are listed in Table SI.

Cell viability assay. To assess cell viability, CAL-27 and SCC-9 cells were seeded in triplicate in 96-well plates at a density of $5 \times 10^{3}$ cells/well in $100 \mu 1$ of culture medium. The cell proliferation index was measured using an MTT assay, which was performed at $0,24,48$ and $72 \mathrm{~h}$ after transfection, respectively. A total of $10 \mu \mathrm{l}$ of MTT reagent $(5 \mathrm{mg} / \mathrm{ml}$; Sigma-Aldrich; Merck KGaA) was added for $3 \mathrm{~h}$. MTT was solubilized using dimethyl sulfoxide (DMSO; Sigma-Aldrich; Merck KGaA) and plates were read at $490 \mathrm{~nm}$ using Tecan Sunrise microplate reader (Tecan F50; Tecan Group, Ltd.).

EdU proliferation assay. To assess cell proliferation, CAL-27 and SCC-9 cells $\left(5 \times 10^{3}\right.$ cells/well) were seeded in 96-well plates. The cells were incubated under standard conditions in complete media. Transfection of the cells was performed the following day. A total of $48 \mathrm{~h}$ after transfection, cell proliferation was detected using the incorporation of EdU with an EdU Cell Proliferation Assay kit (Guangzhou RiboBio Co., Ltd.). Briefly, the cells were incubated with $50 \mu \mathrm{M}$ EdU for $6 \mathrm{~h}$ before fixation, permeabilization and EdU staining, which were performed according to the manufacturer's protocol. The cell nuclei were stained with DAPI (Sigma-Aldrich; Merck KGaA) at a concentration of $1 \mu \mathrm{g} / \mathrm{ml}$ for $20 \mathrm{~min}$. The proportion of the EdU-incorporated cells was determined with fluorescence microscopy (x200).

Clone formation assay. Single cells at low density (52.63 cells per $\mathrm{cm}^{2}$ ) were seeded over a coating of $0.6 \%$ agarose solution containing supplemented media in normal conditions. The cells were allowed to sit for $10 \mathrm{~min}$ at room temperature and were then covered with a $0.3 \%$ agarose solution. Plates were incubated at $37^{\circ} \mathrm{C}$ in a $5 \% \mathrm{CO}_{2}$ humidified atmosphere and media were replaced every 3-4 days. After 15 days, The cells were then immobilized with $4 \%$ paraformaldehyde for 15 min, and soaked in Giemsa stain (G4640, Beijing Solarbio Science \& Technology Co., Ltd.) for $30 \mathrm{~min}$ at room temperature and the cells were then washed twice with ultra-pure water. Images were acquired using a camera [DSC-HX90; SONY (China), Co., Ltd.].

Lung metastasis model. A total of 12 male BALB/C nude mice (5 weeks old), with a weight of 17-19 g were obtained from the Guangdong Medical Laboratory Animal Center, were used in this study. All mice were placed in an environment with $50-60 \%$ humidity and a temperature of $22-24^{\circ} \mathrm{C}$. The mice were given free access to water and food, and a 12-h light/dark cycle was applied. Following 7 days of environmental adaptation, the mice were randomly assigned into two groups; a sh-NC group and a sh-MIR4713HG group, with 6 mice in each group. For the in vivo metastasis assays, MIR4713-knockdown OTSCC cells and OTSCC cells were injected into the caudal veins of $\mathrm{BALB} / \mathrm{C}$ nude mice. Animal health and behavior was monitored every two days. Preliminary experiments verified that OTSCC cells metastasized to the lungs of mice after four weeks, which was in line with the one-month processing time of lung metastasis model reported in most literature $(20,21)$. Therefore, in the present study, the mice were sacrificed after four weeks. The mice were anesthetized by intraperitoneal injection of $1 \%$ sodium pentobarbital $(50 \mathrm{mg} / \mathrm{kg})$ and then subjected to cervical dislocation for euthanasia. The lungs of the mice were separated and fixed with $4 \%$ paraformaldehyde for 7 days at $4^{\circ} \mathrm{C}$, then embedded in paraffin and sectioned at $4-\mu \mathrm{m}$. The sections were subjected to hematoxylin and eosin (H\&E) staining. Paraffin sections were baked at $59^{\circ} \mathrm{C}$ for $30 \mathrm{~min}$, dewaxed in xylene and hydrated in a series of gradient alcohol, followed by immersion in hematoxylin dye solution at room temperature for $5 \mathrm{~min}$ to stain the nuclei, in hydrochloric 
acid for $5 \mathrm{sec}$ for differentiation, and in lithium carbonate to stain the nuclei blue. They were then immersed in eosin at room temperature for $5 \mathrm{~min}$ to stain the cytoplasm. Finally, paraffin sections were observed under an optical microscope at a magnification (x200; Nikon Corporation).

Subcutaneous tumor xenograft assay. A total of 10 male BALB/C nude mice (5 weeks old), with a weight of 17-19 g were obtained from the Guangdong Medical Laboratory Animal Center, were used in this study. All mice were placed in an environment with $50-60 \%$ humidity and a temperature of $22-24^{\circ} \mathrm{C}$. The mice were given free access to water and food, and a 12-h light/dark cycle was applied. Following 7 days of environmental adaptation, the mice were randomly assigned into two groups; a sh-NC group and a sh-MIR4713HG group, with 5 mice in each group. The mice were inoculated subcutaneously in the right flank with $2 \times 10^{6}$ cells. Animal health and behavior was monitored every two days. Tumor growth was observed and measured weekly, and tumors were finally excised according to the schedule. Tumors were harvested for the measurement of volume and weight after 30 days. The mice were anesthetized by intraperitoneal injection of $1 \%$ sodium pentobarbital $(50 \mathrm{mg} / \mathrm{kg})$ and then subjected to cervical dislocation for euthanasia.

Luciferase reporter assay. The fragment of MIR4713HG containing the target sequence of let-7c-5p was amplified via RT-qPCR and then inserted into a pmirGLO vector (Promega Corporation) to form the wild-type MIR4713HG reporter vector (MIR4713HG-WT). An additional expression vector was also constructed by inserting a mutated binding site and was termed MIR4713HG-mutated-type (MIR4713HG-MUT). CAL-27 and SCC-9 cells were seeded into 24-well plates at a density of $5 \times 10^{4}$ cells/well. When the confluency reached $\sim 80 \%$, cells were co-transfected with MIR4713HG-WT or MIR4713HG-MUT and let-7c-5p mimics or the negative control at $37^{\circ} \mathrm{C}$ for $48 \mathrm{~h}$ using Lipofectamine ${ }^{\circledR} 2000$ (Invitrogen; Thermo Fisher Scientific, Inc.), in accordance with the manufacturer's protocol. The relative luciferase activity was then determined using a Dual-Luciferase Reporter assay system (Promega Corporation) according to the manufacturer's protocol. Luciferase activity was normalized to the activity of Renilla.

To detect the direct binding of let-7c-5p to the target gene TMC7, the entire 3'-untranslated region (3'-UTR) of human TMC7 was amplified via PCR using human genomic DNA as a template. The PCR products were inserted into the p-MIR-reporter plasmid (Ambion; Thermo Fisher Scientific, Inc.). The insertion was confirmed as correct by sequencing. To test the binding specificity, the sequences that interact with the let-7c-5p seed sequence were mutated, and the mutant TMC7 3'-UTR was inserted into an equivalent luciferase reporter. For luciferase reporter assays, $2 \times 10^{5}$ cells were cultured in 24-well plates, and each well was transfected with $1 \mu \mathrm{g}$ firefly luciferase reporter plasmid, $1 \mu \mathrm{g} \beta$-galactosidase $(\beta$-gal) expression plasmid (Ambion; Thermo Fisher Scientific, Inc.), and equal amounts (100 pmol) of pre-let-7c-5p, anti-let-7c-5p or the scrambled negative control RNA using Lipofectamine ${ }^{\circledR}$ 2000 at $37^{\circ} \mathrm{C}$. The $\beta$-gal plasmid was used as a transfection control. After $24 \mathrm{~h}$ post-transfection, the cells were assayed using a Dual-Luciferase Reporter assay system (Promega Corporation).

Immunohistochemistry. Samples were fixed in $10 \%$ formalin, embedded in paraffin, and cut into $4-\mu \mathrm{m}$-thick sections. The specimens were deparaffinized in xylene and dehydrated using a graded series of ethanol. The specimens were then heated in Tris-EDTA buffer ( $\mathrm{pH} 9.0$ ) for $15 \mathrm{~min}$ (except for PCNA-stained samples). After antigen retrieval, the specimens were treated with $3 \% \mathrm{H}_{2} \mathrm{O}_{2}$ in methanol for $30 \mathrm{~min}$ at room temperature to inhibit the activity of endogenous peroxidase, and incubated with $10 \%$ goat serum for $20 \mathrm{~min}$ at room temperature to block non-specific binding of the immunoreagents. Samples were incubated at $4^{\circ} \mathrm{C}$ overnight with a primary antibody against E-cadherin (1:300; cat. no. 20874-1-AP; Proteintech Group, Inc.), Ki67 (1:200; ab16667; Abcam) and vimentin (1:300; ab137321; Abcam), followed by incubation with the secondary antibody (code number K4001; Dako EnVision+System-horseradish peroxidase-labelled polymer, anti-mouse; Agilent Technologies, Inc.). The sections were subjected to the hyper-sensitive polymer method, and 4', 6-diamidino-2-phenylindole (DAPI) was used as the chromogen. Images were captured using an optical microscope (x200; Nikon Corporation).

$R T-q P C R$. Total RNA was extracted from CAL-27 and SCC-9 cells using TRIzol ${ }^{\circledR}$ reagent (Invitrogen; Thermo Fisher Scientific, Inc.). RNA quality was assessed using the 260/280 nm absorbance ratio, and concentration was quantified using a microplate reader. Subsequently, isolated RNA was reverse transcribed into cDNA using a PrimeScript RT reagent kit (Takara Bio, Inc.). The conditions of RT were as follows: $38^{\circ} \mathrm{C}$ for $15 \mathrm{~min}$ and $85^{\circ} \mathrm{C}$ for $5 \mathrm{sec}$. RT-qPCR analysis was performed using Maxima SYBR-Green/ROX qPCR Master Mix (Invitrogen; Thermo Fisher Scientific, Inc.). The following thermocycling conditions were used for the RT-qPCR: $95^{\circ} \mathrm{C}$ for $10 \mathrm{~min}, 95^{\circ} \mathrm{C}$ for $15 \mathrm{sec}, 62^{\circ} \mathrm{C}$ for $30 \mathrm{sec}$, and $72^{\circ} \mathrm{C}$ for $30 \mathrm{sec}$. The relative mRNA expression was calculated using the $2^{-\Delta \Delta \mathrm{Cq}}$ method (22), GAPDH and U6 were set as internal controls. Primer sequences are listed in Table SII.

Protein extraction and western blot analysis. OTSCC tissues were homogenized using a Beads crusher. Homogenized tissues and adherent cells were lysed using RIPA buffer (product no. 08714-04; Nacalai Tesque, Inc.). Protein quantification was carried out using a BCA protein assay kit. Proteins $(30 \mu \mathrm{g})$ were separated via $10 \%$ SDS-PAGE and then transferred to polyvinylidene difluoride membranes. The membranes were washed, then blocked with 5\% skimmed milk for $1 \mathrm{~h}$ at room temperature and incubated overnight at $4^{\circ} \mathrm{C}$ with a primary antibody specific for E-cadherin $(1: 1,000$; cat. no. 20874-1-AP; ProteinTech Group, Inc.), N-cadherin (1:1,000; product code ab76011), vimentin $(1: 1,000$; product code ab137321) and TMC7 (1:1,000; product code ab191521; all from Abcam) and GAPDH (1:2,000; product no. 5174S; Cell Signaling Technology, Inc.), followed by incubation with horseradish peroxidase-conjugated secondary antibodies for $60 \mathrm{~min}$ at $37^{\circ} \mathrm{C}$ (both 1:500; product codes ab6789 or ab6721; both from Abcam). Immunocomplexes were visualized using 
an enhanced chemiluminescence assay kit (ECL Plus Western Blotting Detection Reagents; GE Healthcare Life Sciences).

Wound healing assay. Cells $\left(1 \times 10^{3}\right.$ cells/well) were seeded into 6-well plates and grown until $100 \%$ confluent. A scratch was produced with a sterile $200-\mu 1$ pipette tip in the cell monolayer followed by culture in DMEM for an additional $24 \mathrm{~h}$ at $37^{\circ} \mathrm{C}$. Images were captured using an optical microscope (x100; Nikon Corporation), and the migration distance was measured at 0 and $24 \mathrm{~h}$ after scratching using Image J software (version 1.48; National Institutes of Health).

Transwell migration assay. For the detection of migration capacity, CAL-27 and SCC-9 cells $\left(1 \times 10^{4} ; 150 \mu \mathrm{l}\right)$ were collected in serum-free medium and spread onto the upper chamber of a Transwell plate. The lower chamber was filled with $700 \mu \mathrm{l}$ of medium containing $10 \%$ FBS. The plates were incubated at $37^{\circ} \mathrm{C}$ for $24 \mathrm{~h}$. The membranes were then fixed with $4 \%$ methanol at $4^{\circ} \mathrm{C}$, stained with $0.1 \%$ crystal violet for $30 \mathrm{~min}$ at room temperature, and the staining was observed under an optical microscope (x200). For the invasion assay, $80 \mu 1$ Matrigel solution (BD Biosciences) was used to precoat the Transwell membrane for $30 \mathrm{~min}$ at $37^{\circ} \mathrm{C}$.

Statistical analysis. Student's t-test, One-way ANOVA and Spearman's rank correlation coefficient were performed using Graph Pad Prism 8 (GraphPad Software, Inc.). The post hoc test used was Tukey's test. Paired t-test was used for the comparison between the tumor and adjacent non-tumor tissues of the same patients. Experiments were performed in triplicate, with three independent experiments. Data are presented as the mean \pm SEM. $\mathrm{P}<0.05$ was considered to indicate a statistically significant difference.

\section{Results}

lncRNA MIR4713HG is upregulated in OTSCC tissue and cell lines. In order to investigate the potential role of MIR4713HG in OTSCC, the present study first examined the expression pattern of MIR4713HG in OTSCC using the information acquired from online databases (23).

According to the RNA-sequencing data, the expression of MIR4713HG was significantly upregulated in OTSCC tissues compared with normal tissues and its expression was stage-dependent in OTSCC, indicating its potential role in tumor progression (Fig. 1A and B). To investigate the prognostic value of MIR4713HG, the present study extracted the expression data of MIR4713HG from 113 patients with OTSCC.The expression level of MIR4713HG was significantly higher in patients with OTSCC (Fig. 1C). Furthermore, the high expression of MIR4713HG indicated an unfavorable prognosis in OTSCC (Fig. 1D). To confirm the online data, the present study performed RT-PCR in order to determine the expression level of MIR4713HG in 20 paired OTSCC and adjacent non-tumor tissues (Fig. 1E). Additionally, the present study also examined the expression levels of MIR4713HG in multiple OTSCC cell lines (CAL-27, SCC-9, SCC-4, SCC-15 and SCC-25) and HPLF cell line. It was revealed that the expression of MIR4713HG was significantly increased in OTSCC cell lines as well (Fig. 1F). The differential expression of MIR4713HG in OTSCC and adjacent non-tumor tissues suggests that MIR4713HG may have an important role in the progression of OTSCC.

IncRNA MIR4713HG regulates the proliferation and migration of OTSCC cells in vitro. To further investigate the regulating role of MIR4713HG in OTSCC, the present study performed a series of malignancy-associated experiments on CAL-27 and SCC-9 cell lines. First, the present study constructed MIR4713HG-overexpressing/knockdown cell lines via pcDNA-MIR4713HG and sh-MIR4713HG, respectively. The overexpression and knockdown efficiency were evaluated using RT-PCR. The expression of MIR4713HG was significantly upregulated/downregulated in constructed cell lines (Fig. S1A and B). MTT assays were performed to assess the effect of MIR4713HG expression level on OTSCC cell proliferation. After $72 \mathrm{~h}$ of observation, the present study clearly identified that the overexpression of MIR4713HG improved the OTSCC cell proliferation rate, and the administration of sh-MIR4713HG achieved the exact opposite effect (Fig. 2A and B). In the colony formation assay, MIR4713HG-overexpressing OTSCC cells formed more clones on the culture dish after 14 days compared with the control group, while the clonogenicity of OTSCC cells was compromised after MIR4713HG-knockdown (Fig. 2C). In order to ensure the reliability of tumor cell proliferation experiments, EdU assays were used to detect the cell proliferation rate. In the EdU cell proliferation assay, the staining intensity was significantly stronger in MIR4713HG-overexpressing OTSCC cells and weaker in MIR4713HG-knockdown OTSCC cells, confirming the positive effect of MIR4713HG on OTSCC growth (Fig. 2D and E). OTSCC is a highly metastatic cancer. To evaluate the regulatory role of MIR4713HG in cellular migration and metastasis in OTSCC, the present study performed a wound healing assay and Transwell assay. The wound healing results revealed that the overexpression of MIR4713HG accelerated the migration rate of OTSCC cells while the downregulation of MIR4713HG slowed down the migration rate (Fig. 3A). The Transwell assay results indicated that MIR4713HG was a contributing factor in OTSCC metastasis, as overexpression of MIR4713HG improved the invasion rate of OTSCC cells, while the downregulation of MIR4713HG decreased the invasion rate (Fig. 3B and C).

Knockdown of lncRNA MIR4713HG inhibits tumor growth in vivo. To further investigate the impact of MIR4713HG on the tumorigenicity of OTSCC in vivo, 12 nude mice were injected with transfected OTSCC cells (6 sh-MIR4713HG mice and 6 sh-NC mice). At 30 days post-inoculation, tumors were harvested for the measurement of volume and weight (Fig. 4A). The largest tumor was $612.4 \mathrm{~mm}^{3}\left(10.5 \times 10.8 \mathrm{~mm}^{3}\right)$. The volume of tumors in the sh-MIR4713HG group was smaller than that of the sh-NC group (Fig. 4B). The tumor weight was decreased in the sh-MIR4713HG group compared with the sh-NC group (Fig. 4C). The epithelial-mesenchymal transition (EMT) is a crucial process in cancer metastasis $(24,25)$. Therefore, the present study examined the expression levels of EMT-associated proteins, E-cadherin/vimentin in the harvested tumors via immunohistochemistry and western blotting. The knockdown of MIR4713HG increased the expression levels of E-cadherin 


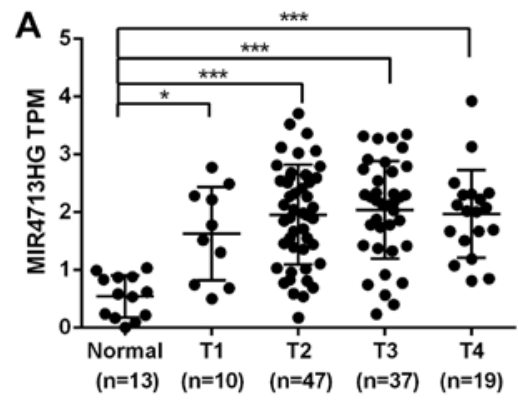

C
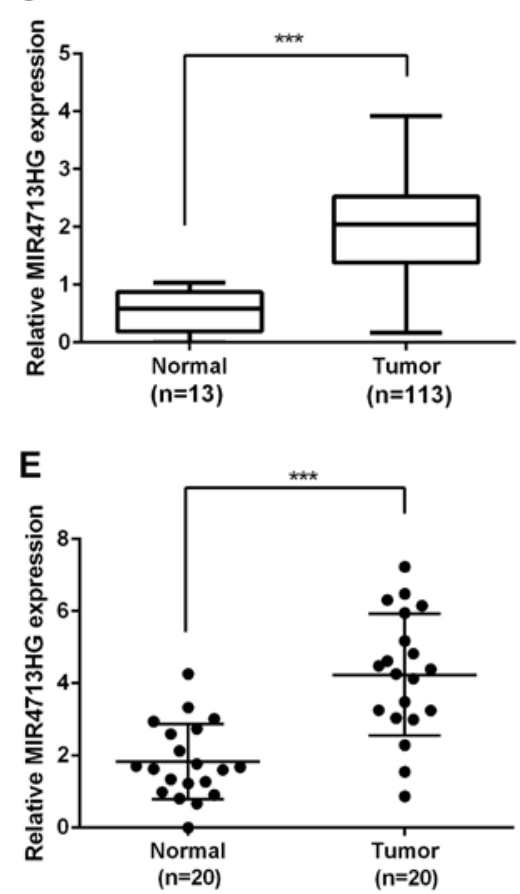

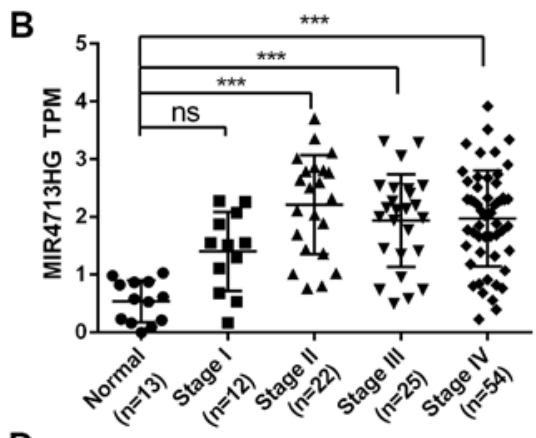

D

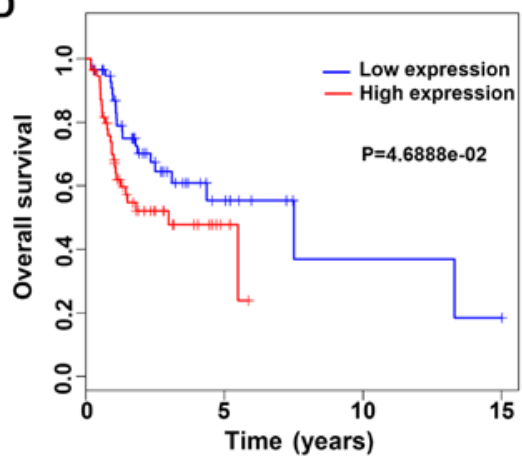

$\mathbf{F}$

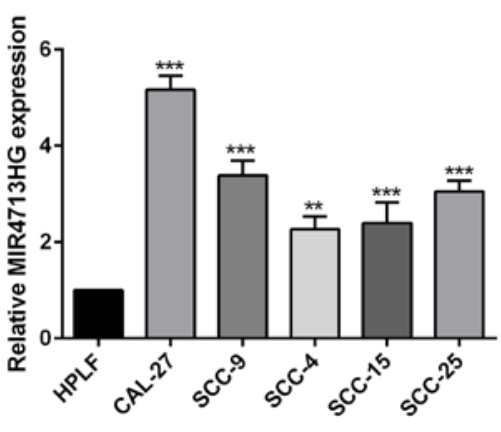

Figure 1. lncRNA MIR4713HG is specifically upregulated in OTSCC tissues and cell lines. (A) The expression of MIR4713HG (TCGA expression data, TPM) in normal oral epithelium tissues compared with OTSCC tissues (T1-T4). (B) The expression of MIR4713HG (TPM data) in normal oral epithelium tissues compared with OTSCC tissues (Stage I-IV). (C) The relative expression level of MIR4713HG in OTSCC and adjacent non-tumor tissues. (D) Survival analysis of patients with OTSCC with high/low expression of MIR4713HG. (E) The relative expression level of MIR4713HG in 20 paired OTSCC and adjacent non-tumor tissues. (F) The relative expression level of MIR4713HG in human OTSCC cell lines (CAL-27, SCC-9, SCC-4, SCC-15 and SCC-25, and the HPLF cell line. Data are presented as the mean \pm SEM. ${ }^{*} \mathrm{P}<0.05,{ }^{* *} \mathrm{P}<0.01$ and ${ }^{* * *} \mathrm{P}<0.001$. OTSCC, oral tongue squamous cell carcinoma; TCGA, The Cancer Genome Atlas; HPLF, human periodontal ligament fibroblast; ns, not significant; TPM, Transcripts Per Kilobase of exon model per Million mapped reads.

and suppressed the expression of vimentin (Fig. 4D and E), suggesting that MIR4713HG affects the metastatic process of OTSCC via accelerating EMT. Furthermore, the present study also constructed lung metastasis models via injecting OTSCC cells into the caudal veins of nude mice. Macroscopically, the mice injected with MIR4713HG-knockdown OTSCC cells exhibited statistically less lung metastasis than the control mice (Fig. 4F-H). In addition, the expression of MIR4713HG was downregulated in the subcutaneous tumor tissue transfected with sh-MIR4173HG (Fig. S1C).

IncRNA MIR4713HG regulates malignant behaviors of OTSCC via binding with miRNA let-7c-5p. IncRNAs commonly act as competing endogenous RNAs to regulate cellular functions. Using publicly available DIANA Tools (26), the present study successfully observed let-7c-5p directly binding with MIR4713HG (Fig. 5A). To test the direct binding of let-7c-5p to MIR4713HG, a luciferase reporter assay was performed as previously described. The administration of let-7c-5p mimics resulted in a $0-20 \%$ reduction of luciferase reporter activity compared with the control group (Fig. 5B). Furthermore, the present study introduced point mutations into the corresponding sites in MIR4713HG to eliminate the predicted let-7c-5p binding sites. This mutated luciferase reporter was unaffected by the overexpression of let-7c-5p (Fig. 5B). According to the RT-PCR results, the expression of let-7c-5p increased after MIR4713HG knockdown and decreased after MIR4713HG overexpression (Fig. 5C). Based on The Cancer Genome Atlas (TCGA) database (23), the expression of let-7c-5p was downregulated in OTSCC tumors and its expression pattern was confirmed in 20 paired OTSCC tumor and adjacent nontumor tissues (Fig. S2A and B). The expression of let-7c-5p was negatively correlated with MIR4713HG (Fig. S2C and D). CAL-27/SCC-9 cells were transfected with let-7c-5p mimics and inhibitor, and the expression of let-7c-5p was significantly upregulated/downregulated in constructed cell lines (Fig. S2E and F). To assess the effect of let-7c-5p expression levels on OTSCC cell proliferation, the present study performed rescue experiments via 
A

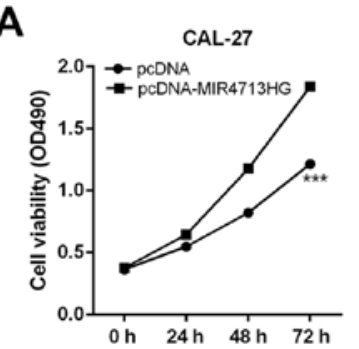

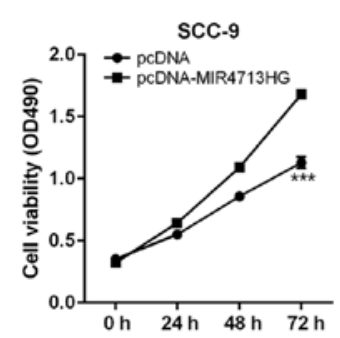
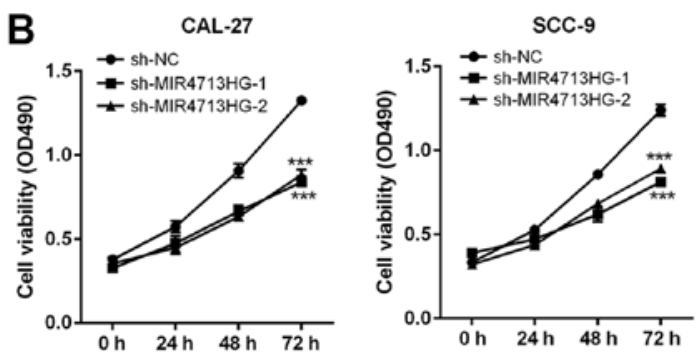

C
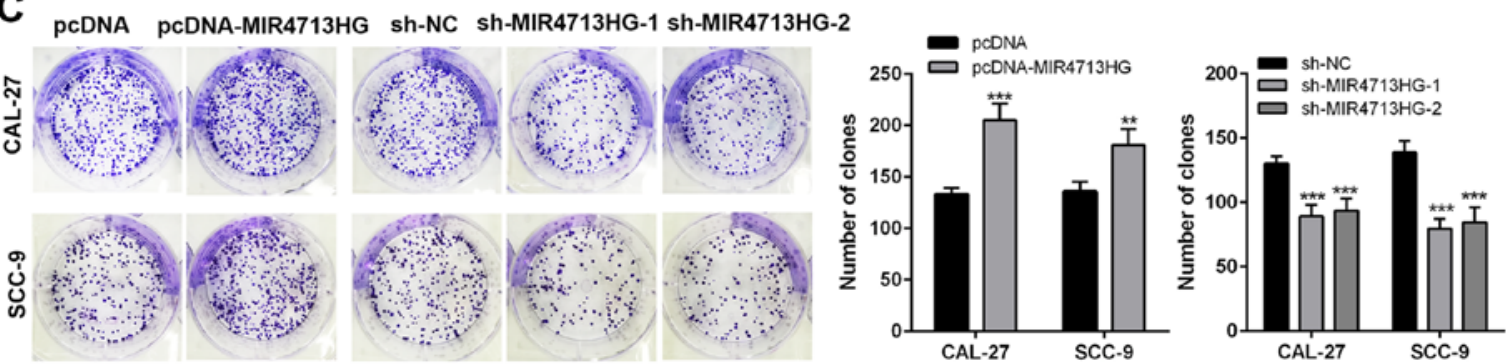

D
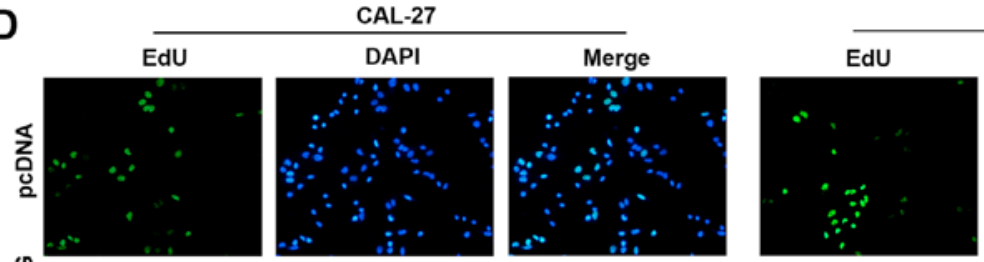

SCC-9
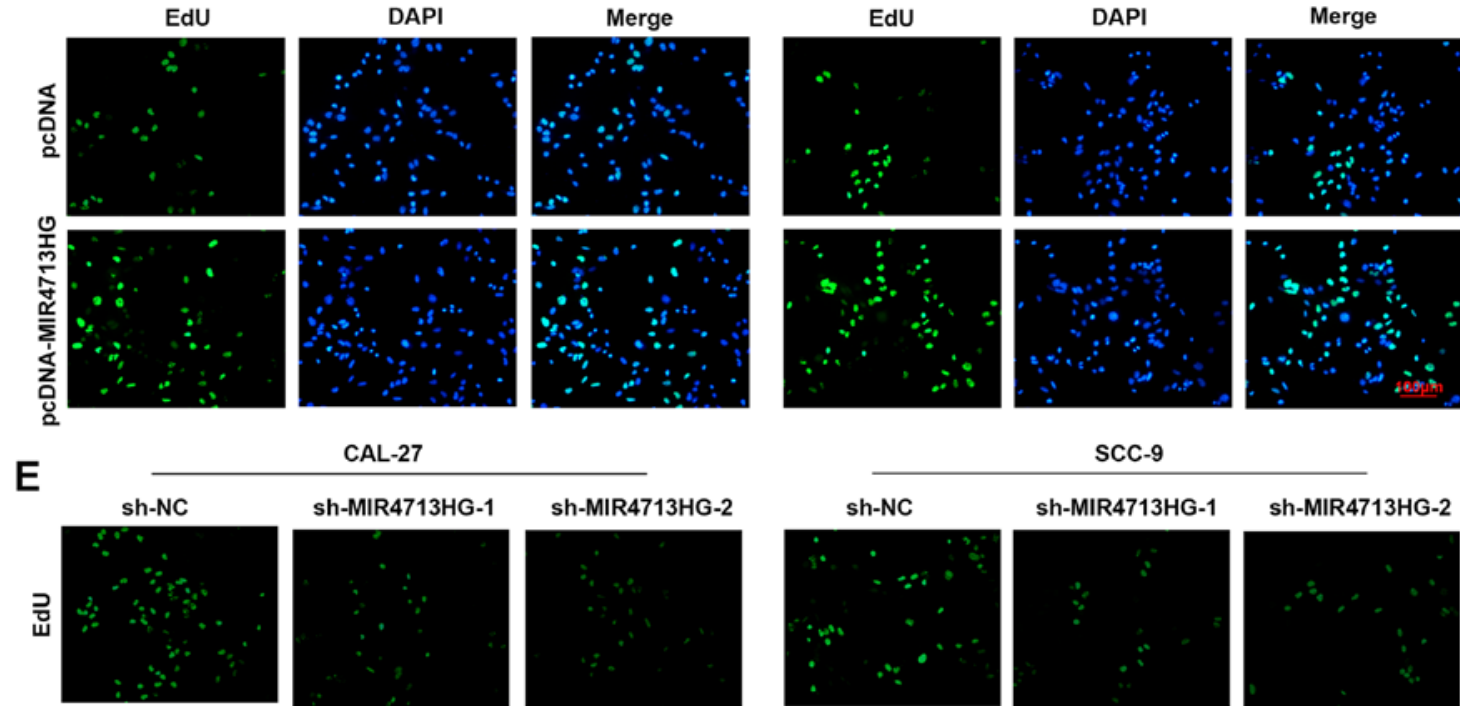

CAL-27
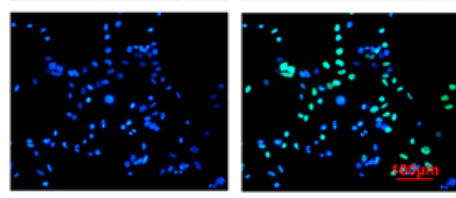

scC-9
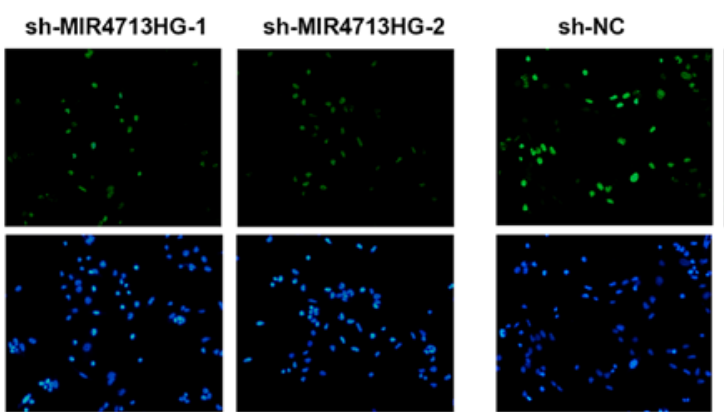

sh-MIR4713HG-1

sh-MIR4713HG-2
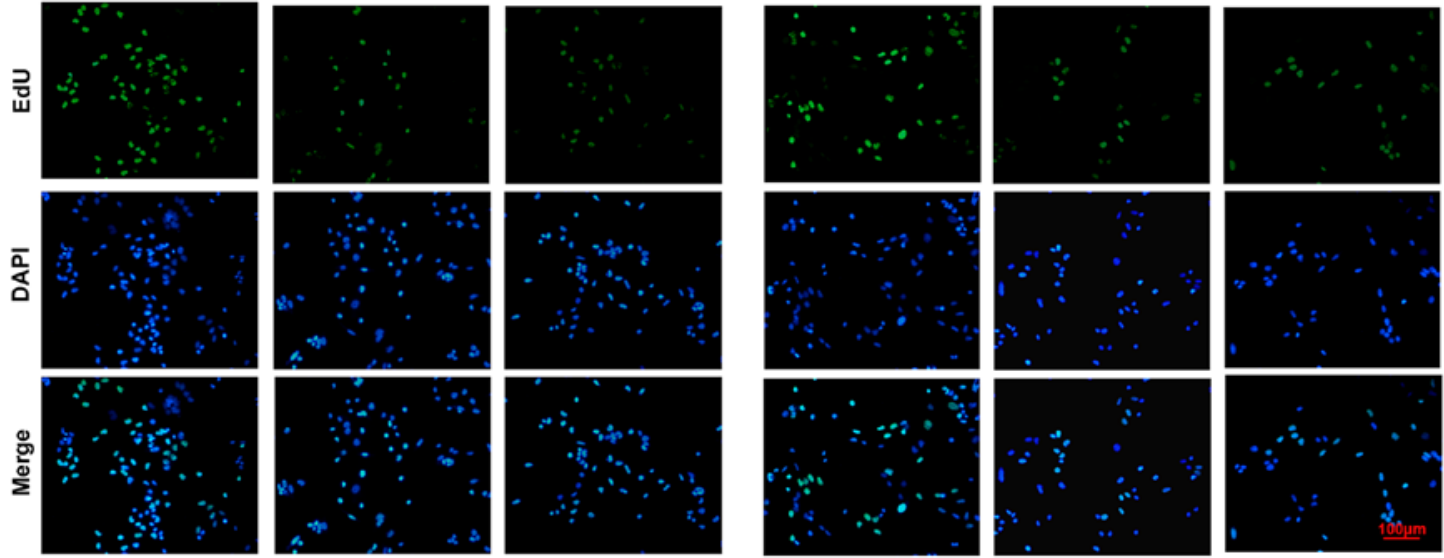

Figure 2. IncRNA MIR4713HG modulates growth of OTSCC. (A) The relative growth rates were measured using MTT analysis and compared between 2 groups at the indicated time-points $(0,24,48$ and $72 \mathrm{~h}$ ) in two different cell lines transfected with pcDNA-MIR4713HG or control. (B) The relative growth rates were measured using MTT assay and compared between two groups at the indicated time-points $(0,24,48$ and $72 \mathrm{~h})$ in two different cell lines transfected with sh-MIR4713HG or control. (C) Colony formation of 2 different cell lines transfected with different treatments. (D and E) Immunofluorescence images of EdU staining intensity of CAL-27/SCC-9 cell lines transfected with sh-MIR4713HG or pcDNA-MIR4713HG. Data are presented as the mean \pm SEM. ${ }^{* *} \mathrm{P}<0.01$ and ${ }^{* * *} \mathrm{P}<0.001$. OTSCC, oral tongue squamous cell carcinoma; sh-, short hairpin; NC, negative control.

MTT and clone formation assays. MIR4713HG overexpression significantly improved cell proliferation and let-7c-5p mimics suppressed the cell viability and clonogenicity compared with the NC group. The let-7c-5p mimics partially rescued the stimulative effects of MIR4713HG overexpression on OTSCC cell proliferation (Fig. 5D and E). The present study also performed rescue experiments via Transwell assays. The addition of let-7c-5p mimics partially rescued the improved metastatic potential of OTSCC cells induced by MIR4713HG overexpression compared with pcDNA-MIR4713HG group (Fig. 5F).

TMC7 is the downstream target gene of miRNA let-7c-5p. To identify the downstream target of let-7c-5p, the present study used online bioinformatics platforms, DIANA tools 

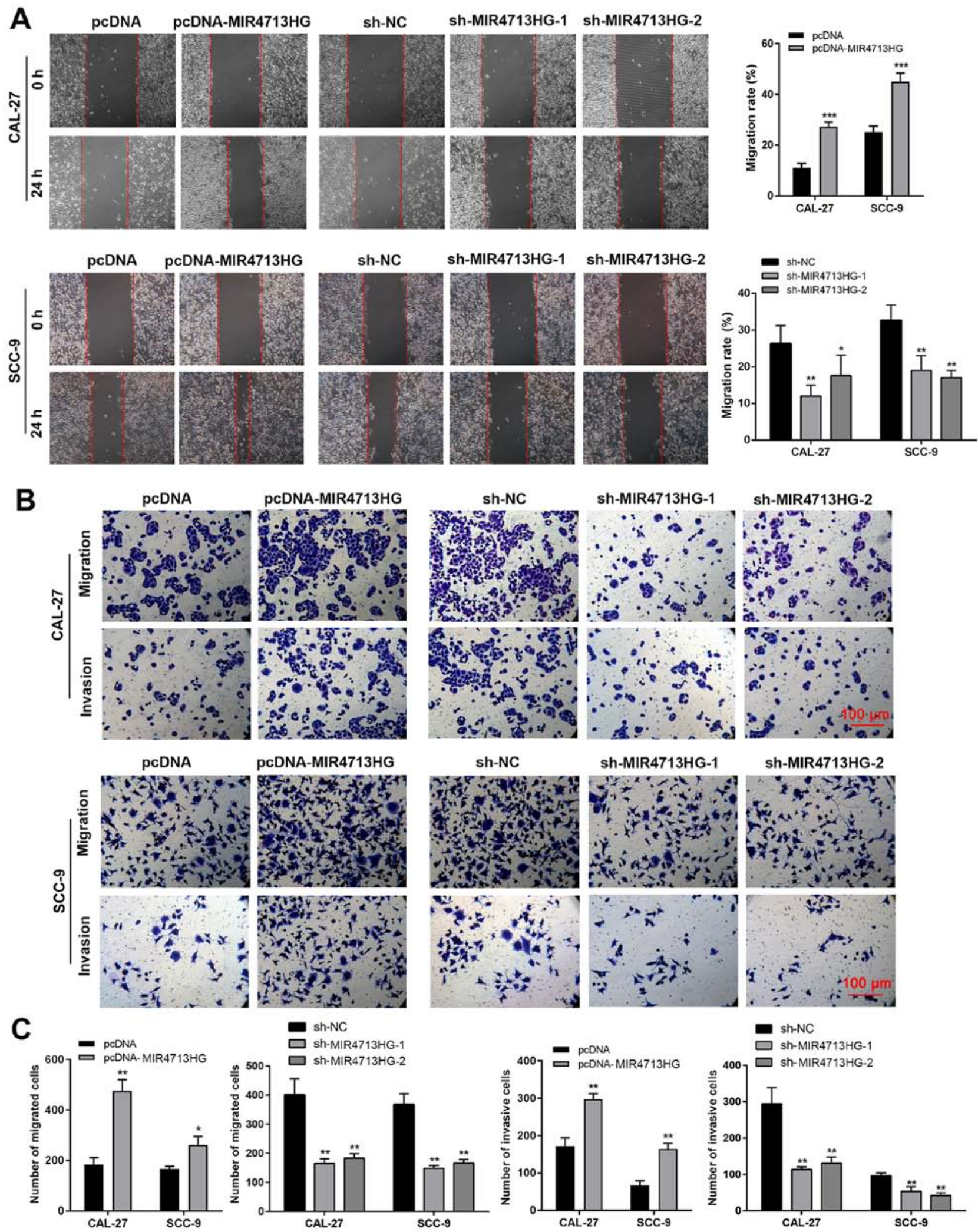

Figure 3. IncRNA MIR4713HG modulates the migration and invasion of OTSCC cells. (A) Wound healing assays were performed to evaluate the migration ability of OTSCC cells after transfection with sh-MIR4713HG or pcDNA-MIR4713HG. Images were captured at 0 and $24 \mathrm{~h}$. The migration rate data are presented as the mean \pm SEM. (B and C) Transwell assay was performed to evaluate the metastasis ability of OTSCC cells after transfection with sh-MIR4713HG or pcDNA-MIR4713HG. The migration/invasion number data are presented as the mean \pm SEM. ${ }^{*} \mathrm{P}<0.05,{ }^{* * *} \mathrm{P}<0.01$ and ${ }^{* * *} \mathrm{P}<0.001$. OTSCC, oral tongue squamous cell carcinoma; sh-, short hairpin; NC, negative control.

and TargetScan to predict the potential target genes of let-7c-5p $(26,27)$. By overlapping the aforementioned results with OTSCC-overexpressing genes, a list of 79 genes was obtained (Fig. 6A). Among the 79 genes, TMC7 was found to directly bind with let-7c-5p (Fig. 6B). According to the results of the luciferase reporter assay, the administration of let- $7 c-5 p$ 
A

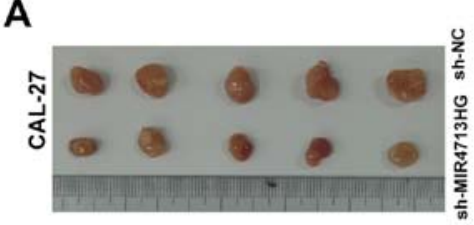

D
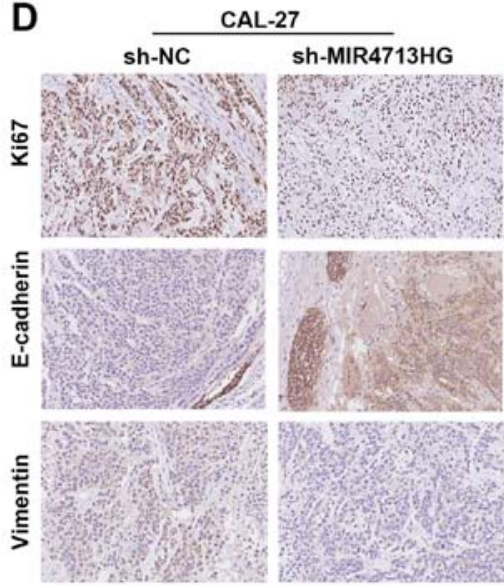

G

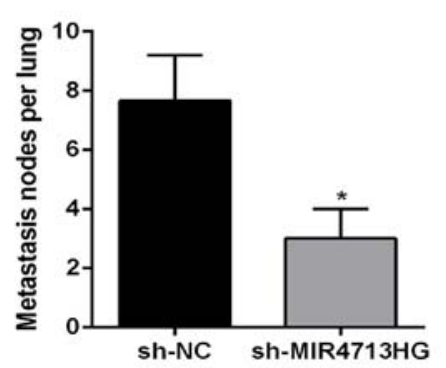

B
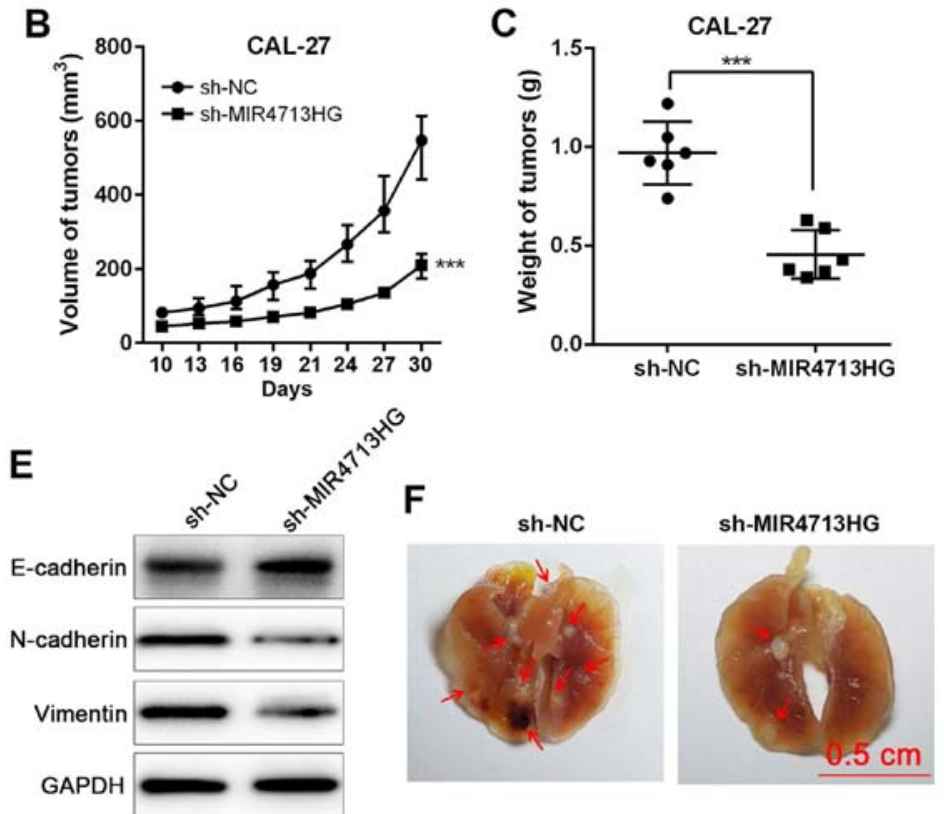

H

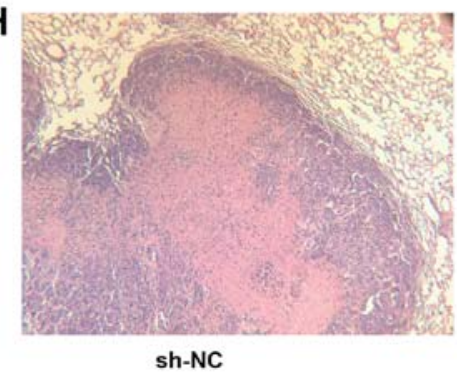

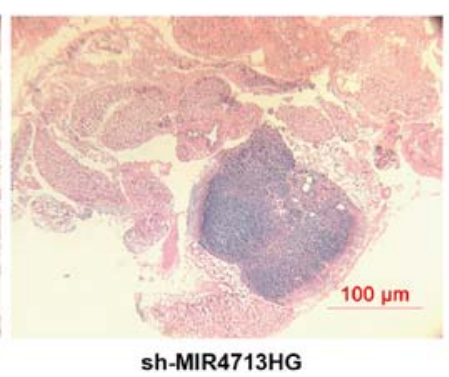

Figure 4. Downregulation of lncRNA MIR4713HG suppresses OTSCC tumor growth and metastasis in vivo. (A) The macroscopic images of xenografted tumors harvested from two groups (sh-MIR4713HG, sh-NC). (B) The volume of xenografted tumors from different groups were compared. The comparison was made at the indicated time-points. Data are presented as the mean \pm SEM. The largest tumor was $612.4 \mathrm{~mm}^{3}\left(10.5 \times 10.8 \mathrm{~mm}^{3}\right)$. $(\mathrm{C}) \mathrm{The}$ weight of tumors harvested from two groups. (D) The immunohistochemical images of paraffin-embedded tumor sections stained with Ki-67/E-cadherin/vimentin. (E) Western blotting was performed to detect the protein level of $\mathrm{N}$-cadherin/E-cadherin/vimentin in CAL-27 cells transfected with sh-MIR4713HG or sh-NC. (F) The macroscopic images of lungs harvested from two groups of lung metastasis models. (G) The number of metastatic lesions was presented as the mean \pm SEM. $(\mathrm{H})$ The microscopic images of hematoxylin and eosin staining of lung tissues. Data are presented as the mean $\pm \mathrm{SEM}$ ( $\mathrm{n}=6 \mathrm{for}$ each group). ${ }^{*} \mathrm{P}<0.05$ and ${ }^{* * *} \mathrm{P}<0.001$. OTSCC, oral tongue squamous cell carcinoma; sh-, short hairpin; NC, negative control.

mimics resulted in a reduction of luciferase reporter activity compared with the control group, and the introduction of point mutations into TMC7 eliminated the predicted let-7c-5p binding sites (Fig. 6C), confirming the direct binding between let-7c-5p and TMC7. Based on TCGA database, the expression of TMC7 was upregulated in OTSCC tumors and its expression pattern was confirmed in 20 paired OTSCC tumor and adjacent non-tumor tissues (Fig. 6D). The same expression pattern of TMC7 was also confirmed at the protein level (Fig. 6E). The expression of TMC7 was negatively associated with let-7c-5p (Fig. 6F). Furthermore, the addition of let-7c-5p mimics significantly suppressed the expression of TMC7 while the addition of let-7c-5p inhibitor greatly boosted the expression of TMC7 (Fig. 6G).

IncRNA MIR4713HG performs its regulatory roles by affecting the let-7c-5p/TMC7 axis. In order to investigate the regulation of MIR4713HG on TMC7 expression, the present study performed RT-qPCR. The overexpression of MIR4713HG significantly increased the expression of TMC7, while the knockdown of MIR4713HG suppressed the expression of TMC7 and both effects were counteracted by let-7c-5p mimics and inhibitor, respectively (Fig. 7A). Based on TCGA database, the expression of TMC7 was positively correlated with MIR4713HG in OTSCC tumors and its expression pattern was confirmed in 20 paired OTSCC tumor and adjacent non-tumor tissues (Fig. 7B and C). The knockdown efficiency was evaluated using RT-qPCR, and the expression of TMC7 was significantly downregulated in constructed cell lines (Fig. S2G). In order to assess the effect of TMC7 expression level on OTSCC cell proliferation, the present study performed rescue experiments via MTT, clone formation and EdU cell proliferation assays. It was revealed that the knockdown of TMC7 could reverse the improved cell proliferation and clonogenicity in OTSCC induced by MIR4713HG overexpression (Fig. 7D-F). In the wound healing assay, the improved cell migration ability induced by MIR4713HG overexpression was counteracted by TMC7 knockdown (Fig. 7G and H). In addition, according to the western blotting results, the overexpression of MIR4713HG accelerated the EMT process in OTSCC, while TMC7 knockdown reversed this effect (Fig. 7I). 
A

Binding site:chr15:51037544-51037557

MIR4713HG WT 5'UCCCUAGUGAGAUGAACUGgGUACCUCA

let-7c-5p 3'

|I|

||||||

UUGgUAUGUUGGaUgaUgGaGU

MIR4713HG MUT 5'UCCCUAGUGAGAUGAACUGGGUACCUCA

C

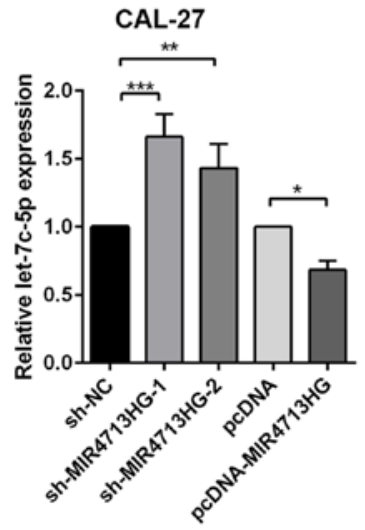

E

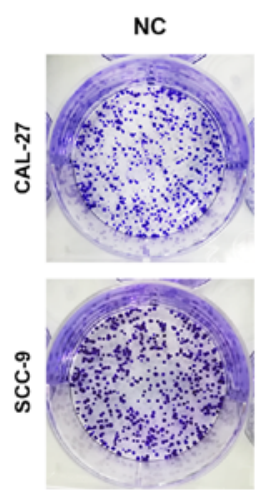

PCDNA-MIR4713HG

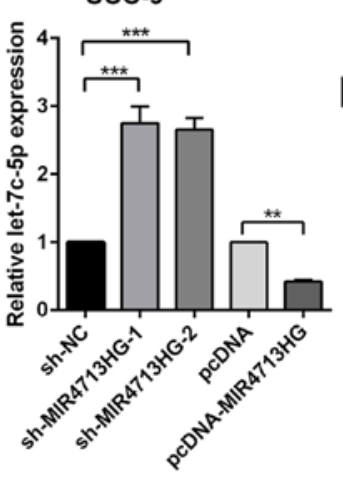

D

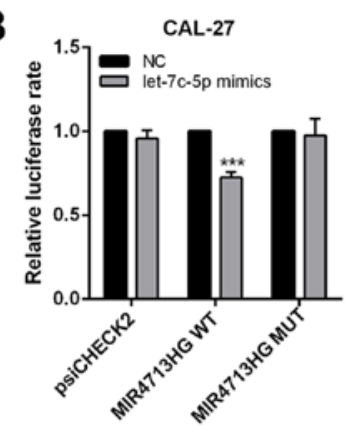

D $\rightarrow \mathrm{NC} \quad \mathrm{CAL}-27$

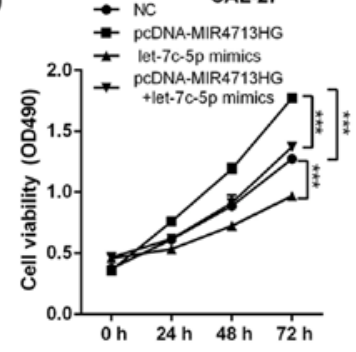

PcDNA-MIR4713HG

+let-7c-5p mimics

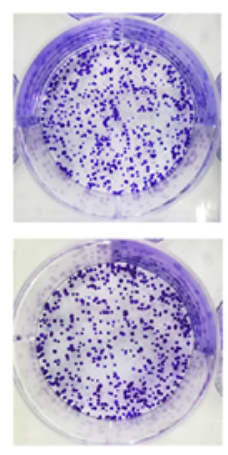

F
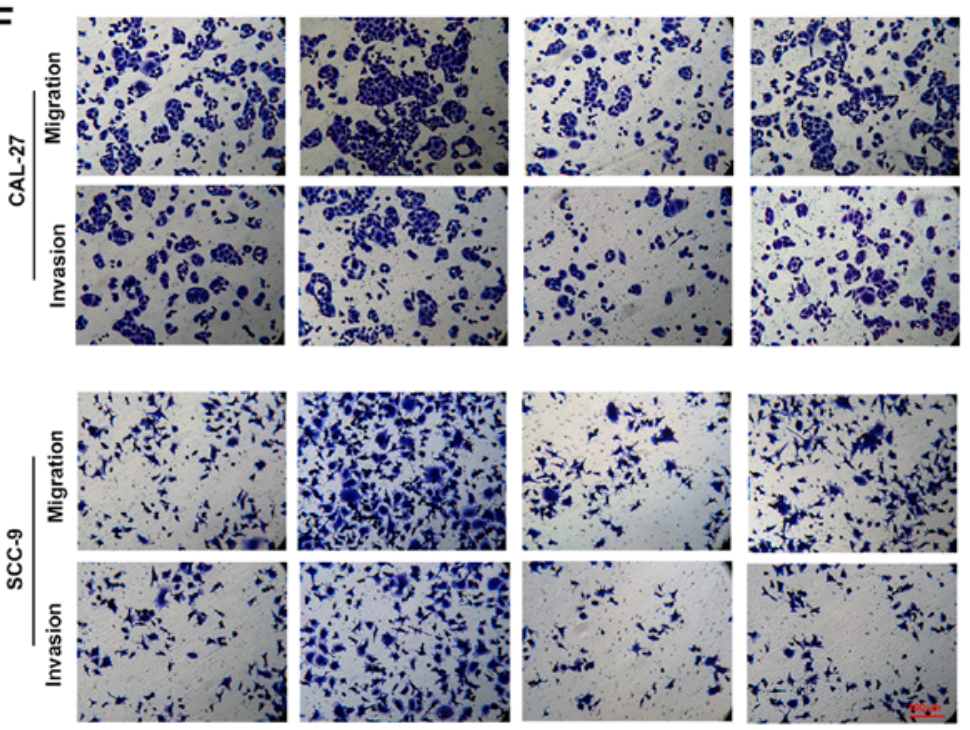

可 ${ }^{\mathrm{NCDNAMMRR} 4713 \mathrm{HG}}$

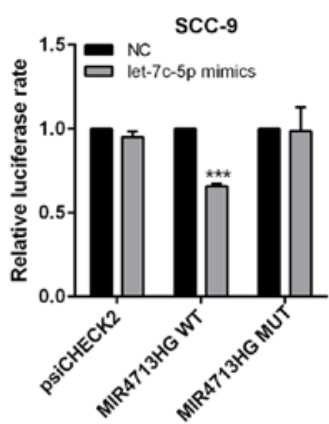

SCC-9

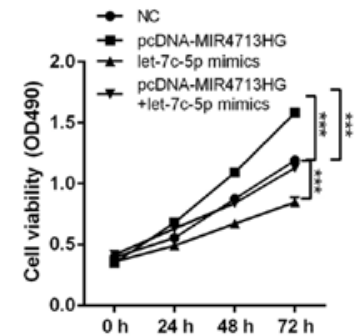

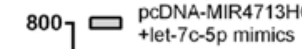

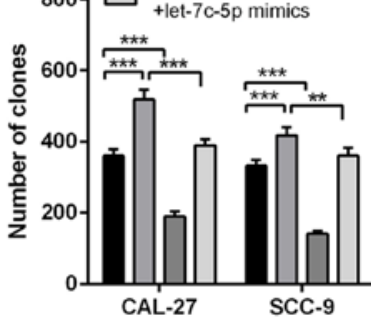

NC

$\square$ pCDNA-MIR4713HG

$\square$ let-7c-5p mimics

$\square \begin{aligned} & \text { pcDNA-MIR4713HG } \\ & + \text { let-7c-5p mimics }\end{aligned}$
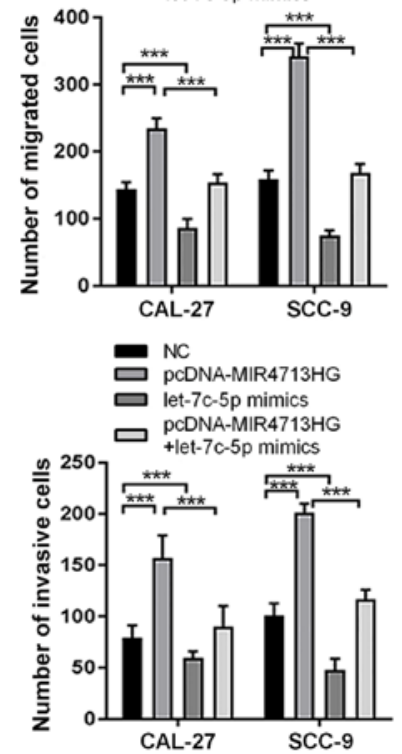

Figure 5. 1ncRNA MIR4713HG binds to let-7c-5p and suppresses its expression. (A) Bioinformatics prediction of the binding site of MIR4713HG and let-7c-5p. The mutation site was marked in red. (B) Dual-luciferase assays were used to detect the luciferase activity in CAL-27/SCC-9 cells transfected with psiCHECK2, MIR4713HG WT or MIR4713HG MUT in the presence of let-7C-5p mimics. (C) The expression of let-7c-5p in CAL-27/SCC-9 cells transfected with different treatments was examined by RT-qPCR. Expression of let-7c-5p in CAL-27/SCC-9 cells transfected with let-7c-5p mimics or inhibitor was examined by RT-qPCR. (D) The relative growth rates were measured using MTT analysis and compared between 3 groups at indicated time-points ( 0,24 , 48 and $72 \mathrm{~h}$ ) in CAL-27/SCC-9 cell lines transfected with pcDNA-MIR4713HG or let-7c-5p mimics or pcDNA-MIR4713HG + let-7c-5p mimics. (E) Clone formation assay of CAL-27/SCC-9 cell lines transfected with pcDNA-MIR4713HG or let-7c-5p or pcDNA-MIR4713HG + let-7c-5p. (F) Transwell assays were performed to evaluate the metastatic abilities of OTSCC cells after transfection with pcDNA-MIR4713HG or let-7c-5p or pcDNA-MIR4713HG + let-7c-5p. Data are presented as the mean $\pm \mathrm{SEM} .{ }^{*} \mathrm{P}<0.05,{ }^{* * *} \mathrm{P}<0.01$ and ${ }^{* * * *} \mathrm{P}<0.001$. WT, wild-type; MUT, mutated; RT-qPCR, reverse transcription-quantitative PCR; sh-, short hairpin; NC, negative control. 

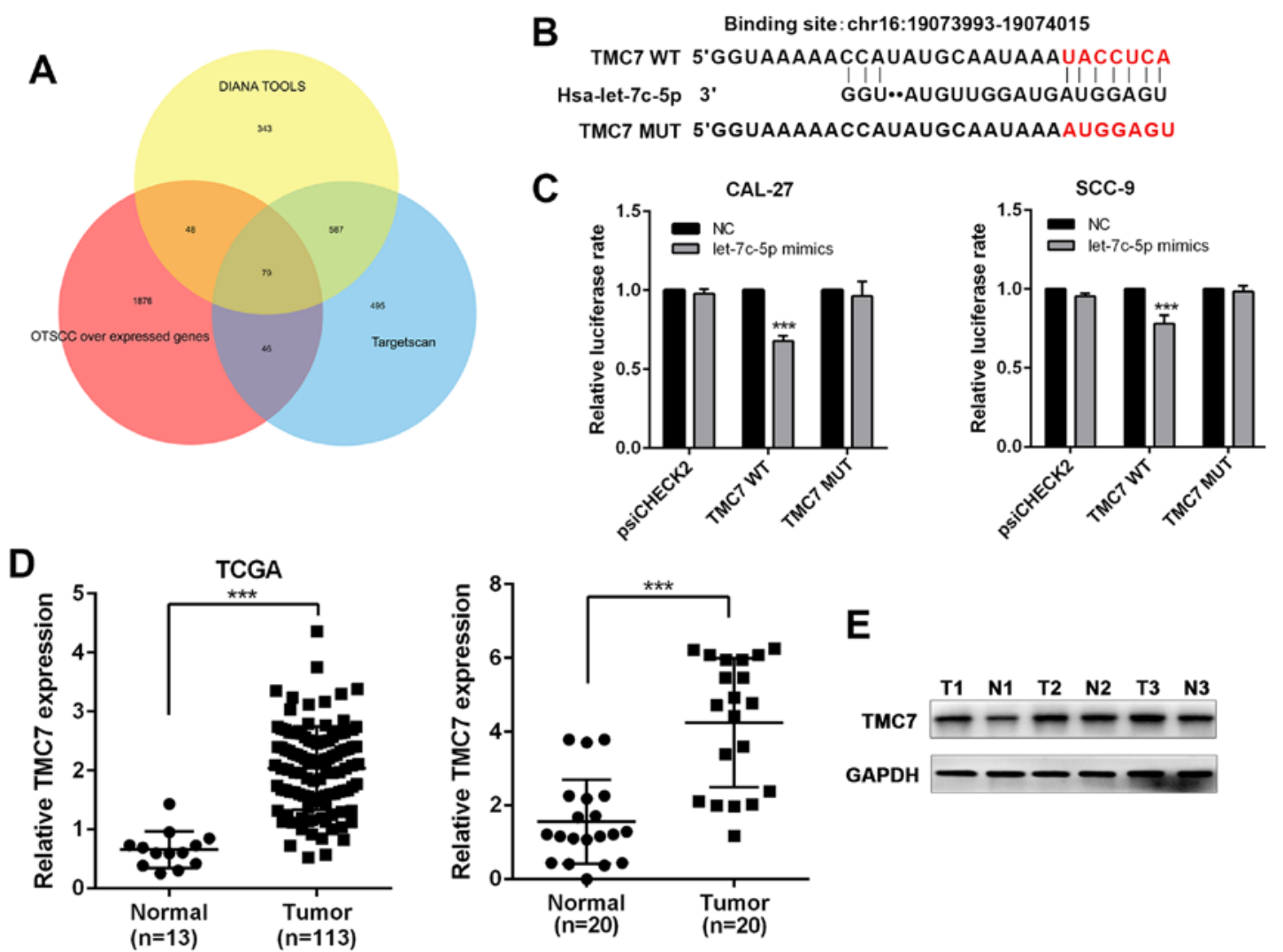

$\mathbf{E}$

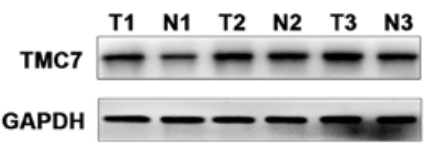

$\mathbf{F}$

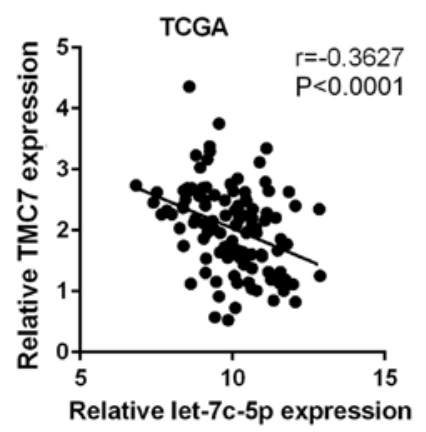

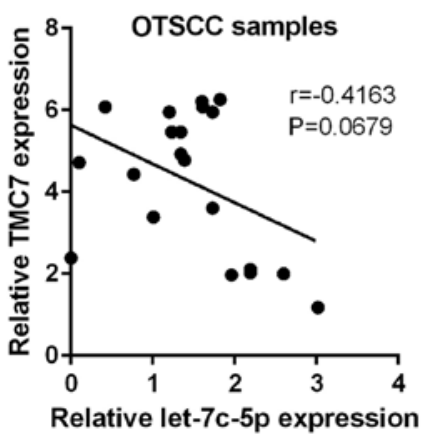

$\mathbf{G}$
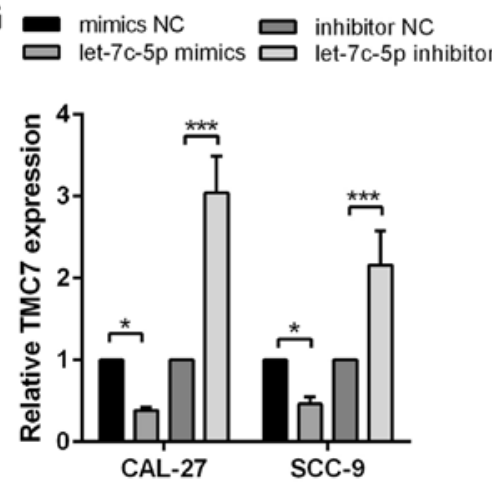

Figure 6. miRNA let-7c-5p suppresses TMC7 in OTSCC cells. (A) A Venn diagram revealed the overlapped genes among OTSCC overexpressed genes, using DIANA Tools and TargetScan. (B) The binding site between let-7c-5p and TMC7. (C) Dual-luciferase assays were used to detect the luciferase activity in CAL-27/SCC-9 cells transfected with psiCHECK2, TMC7 WT or TMC7 MUT in the presence of let-7C-5p mimics. (D) The expression level of TMC7 in OTSCC tissues and adjacent non-tumor tissues based on TCGA data and in 20 paired OTSCC and adjacent non-tumor tissues. (E) Western blotting detection of the expression of TMC7 in 3 paired OTSCC (T) and adjacent non-tumor tissues (N). (F) Spearman analysis of the correlation between the expression of TMC7 and let-7c-5p based on TCGA data and in 20 paired OTSCC and adjacent non-tumor tissues. (G) The expression of TMC7 in CAL-27/SCC-9 cells transfected with let-7c-5p inhibitor or let-7c-5p mimics was examined by RT-qPCR. Data are presented as the mean \pm SEM. ${ }^{*} \mathrm{P}<0.05$ and ${ }^{* * *} \mathrm{P}<0.001$. TMC7, transmembrane channel like 7; OTSCC, oral tongue squamous cell carcinoma; WT, wild-type; MUT, mutated; TCGA, The Cancer Genome Atlas; RT-qPCR, reverse transcription-quantitative PCR; NC, negative control.

\section{Discussion}

Globally, head and neck squamous cell carcinoma (HNSCC) is one of the most common types of cancer and the number of cases of this disease increases by 500,000 annually (28). The therapeutic strategies of oral tongue squamous cell carcinoma include surgical resection, chemotherapy, radiotherapy and targeted therapy. Although great improvements have been made in all of these fields, the prognosis of patients with OTSCC remains unsatisfactory $(29,30)$. Local invasion and metastasis account for the poor prognosis of patients with OTSCC (31). Thus, OTSCC remains a challenging field in cancer and the underlying molecular mechanisms require further investigation.

Long non-coding RNAs and microRNAs are two subtypes of non-coding RNAs with different lengths. It has been well documented that IncRNAs can act as endogenous competing RNAs that interact with miRNAs and participate in human diseases, such as cancer $(32,33)$. In OTSCC, the potential regulating role of lncRNAs remains unclear, except for one IncRNA, HOTTIP, which has been reported to act as an oncogenic factor in OTSCC (17). The present study first revealed that MIR4713HG was upregulated in OTSCC tissues and exhibited itself as an unfavorable prognostic 
A

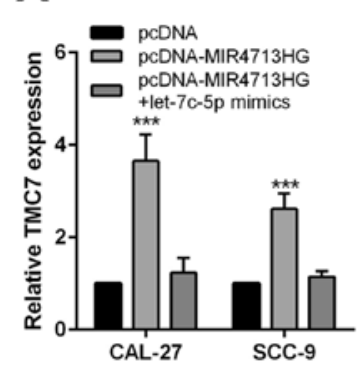

B

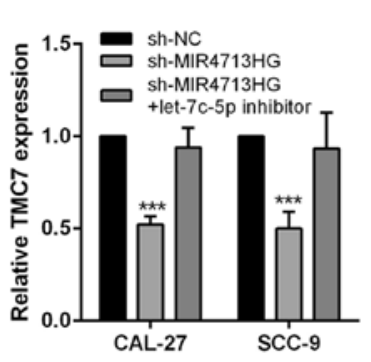

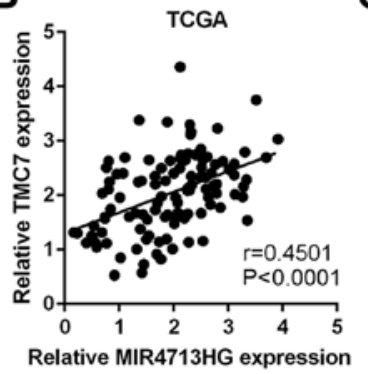

C

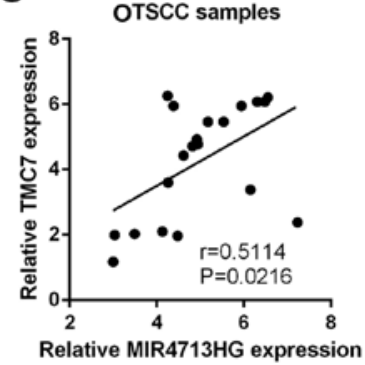

D

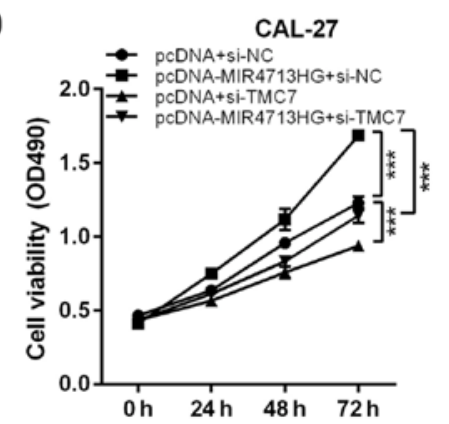

F

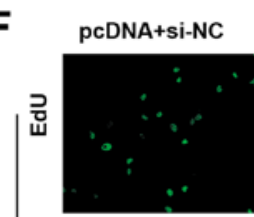

ণิ
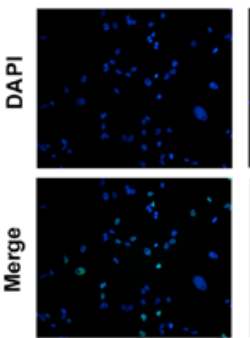

G
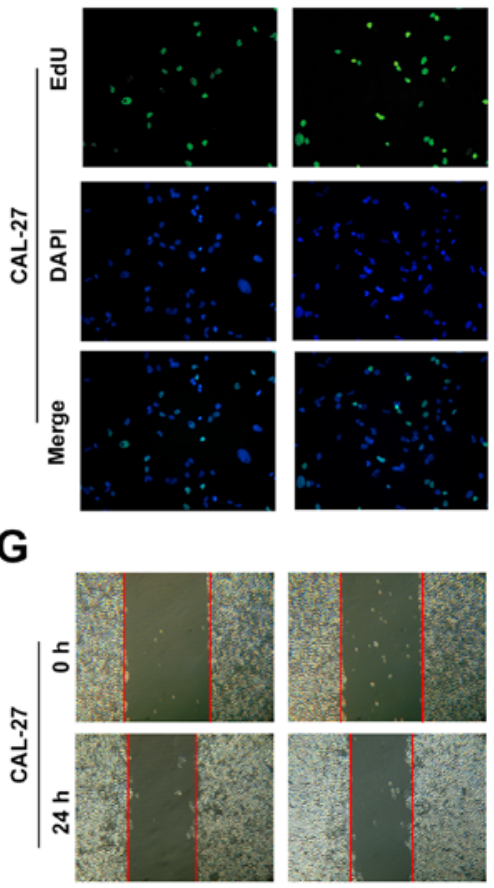

E

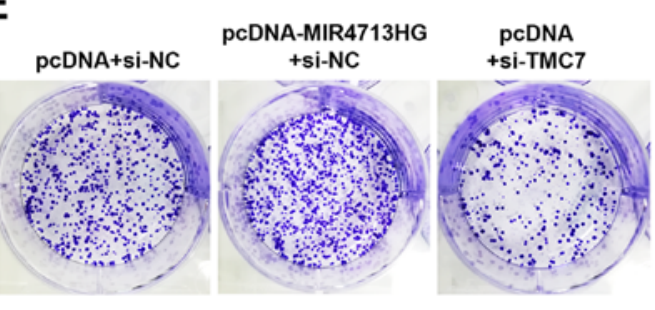

H
pcDNA-MIR4713HG +si-TMC7

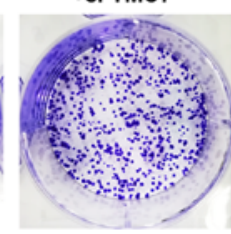

CAL-27
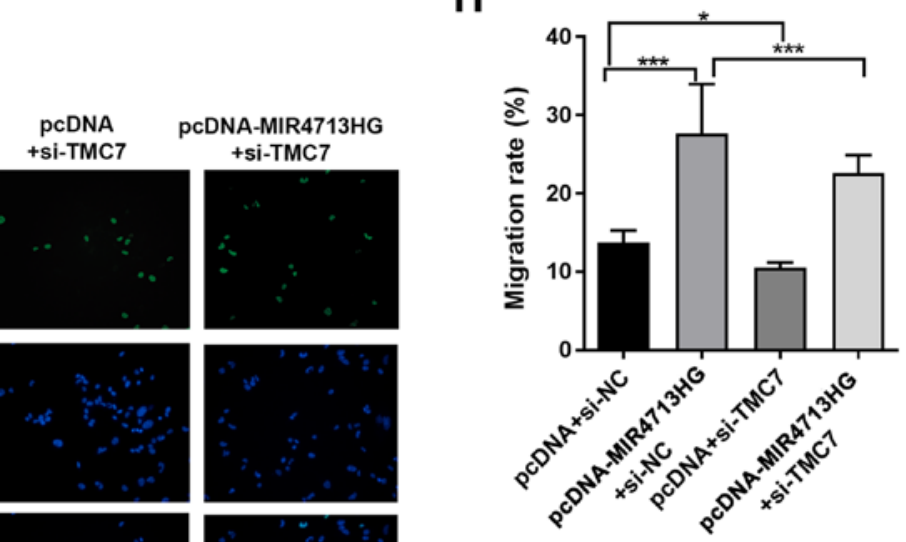

PcDNA-MIR4713HG + si-TMC7
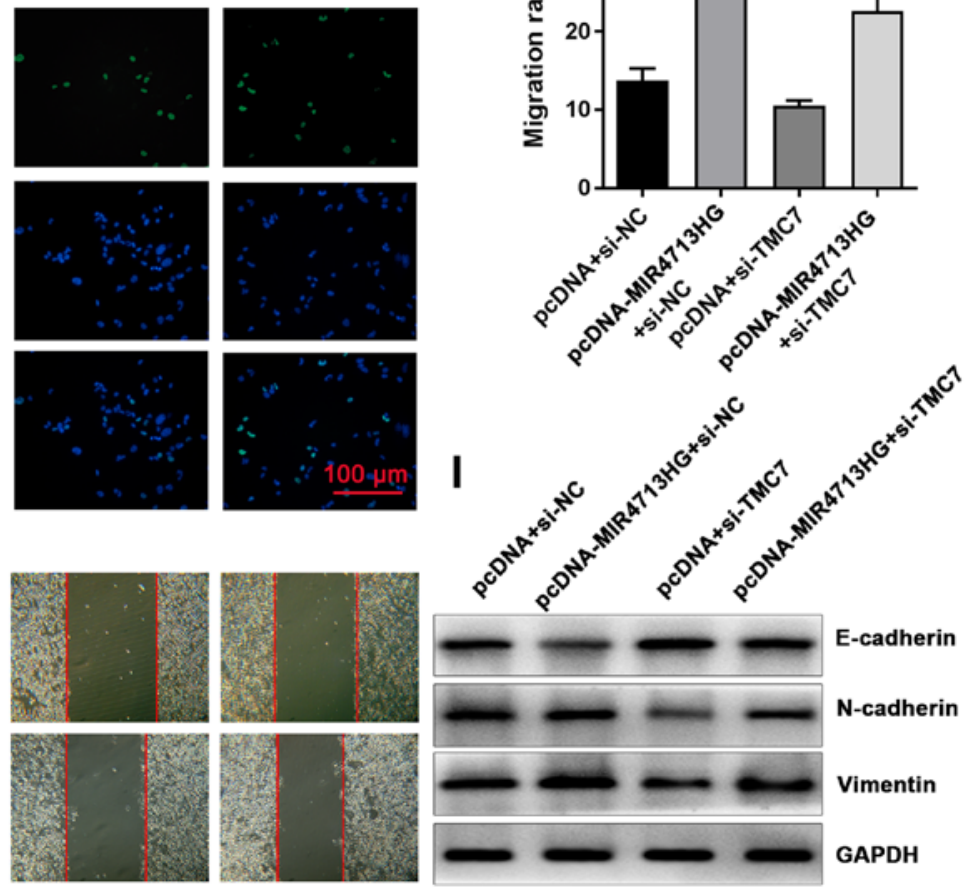

Figure 7. IncRNA MIR4713HG promotes TMC7-mediated proliferation and metastasis through let-7c-5p sponging in vitro. (A) The expression of TMC7 in CAL-27/SCC-9 cells transfected with pcDNA-MIR4713HG/pcDNA-MIR4713HG + let-7c-5p mimics/control or sh-MIR4713HG/sh-MIR4713HG + let-7c-5p inhibitor/sh-NC was examined by RT-qPCR. (B and C) Spearman analysis of the correlation between the expression of lncRNA MIR4713HG and TMC7 based on TCGA data and in 20 paired OTSCC and adjacent non-tumor tissues. (D) The relative growth rates were measured using MTT analysis and compared between 4 groups at indicated time-points $(0,24,48$ and $72 \mathrm{~h})$ in CAL-27/SCC- 9 cell lines transfected with pcDNA + si-NC/pcDNA-MIR4713HG + si-NC/pcDNA + si-TMC7/pcDNA-MIR4713HG + si-TMC7. (E) Clone formation assay of CAL-27/SCC-9 cell lines transfected with pcDNA + si-NC/pcDNA-MIR4713HG + si-NC/pcDNA + si-TMC7/pcDNA-MIR4713HG + si-TMC7. (F) Immunofluorescence images of EdU staining intensity of CAL-27/SCC-9 cell lines transfected with pcDNA + si-NC/pcDNA-MIR4713HG + si-NC/pcDNA + si-TMC7/pcDNA-MIR4713HG + si-TMC7. (G and H) Wound healing assay was performed to evaluate the migration ability of OTSCC cells after transfection with pcDNA + si-NC/pcDNA-MIR4713HG + si-NC/pcDNA + si-TMC7/pcDNA-MIR4713HG + si-TMC7. Images were captured at 0 and $24 \mathrm{~h}$. (I) Western blotting was performed to detect the protein levels of $\mathrm{N}$-cadherin/E-cadherin/vimentin in CAL-27 cells transfected with pcDNA + si-NC/pcDNA-MIR4713HG + si-NC/pcDNA + si-TMC7/pcDNA-MIR4713HG + si-TMC7. Data are presented as the mean \pm SEM. ${ }^{*}<<0.05$ and ${ }^{* * * *} \mathrm{P}<0.001$. TMC7, transmembrane channel like 7; sh-, short hairpin; NC, negative control; si-, small interfering; OTSCC, oral tongue squamous cell carcinoma.

factor in OTSCC patients. To further investigate the function of MIR4713HG, the present study performed gain- or loss-of-function experiments by transfecting OTSCC cell lines with MIR4713HG-overexpressing plasmid or sh-MIR4713HG. The results revealed that MIR4713HG positively regulated the proliferation and clonogenicity of OTSCC cells. Furthermore, 
in the wound healing and Transwell assays, it was revealed that the upregulation of MIR4713HG was correlated with improved migration and metastasis of OTSCC cells.

To test the tumor regulating role of MIR4713HG in vivo, the present study constructed xenograft tumor mouse models and lung metastasis mouse models. Notably, the knockdown of MIR4713HG significantly slowed down the process of tumor growth and suppressed the progression of tumor metastasis in OTSCC in vivo. OTSCC cells have strong invasion and migration capabilities, and they experience enhanced invasion and migration capabilities during EMT, which is an important step in tumor metastasis and spread $(29,30)$. EMT is a multi-step process in which epithelial cells acquire a mesenchymal phenotype, which is characterized by increased exercise capacity, downregulation of epithelial proteins including E-cadherin and $\alpha$-catenin, and overexpression of mesenchymal phenotype proteins, such as vimentin and $\mathrm{N}$-cadherin (34). The present study revealed that knockdown of MIR4713HG upregulated E-cadherin expression and downregulated the expression of $\mathrm{N}$-cadherin and vimentin, which indicated that knockdown of MIR4713HG significantly inhibited EMT. With further investigation, the present study successfully identified that let-7c-5p physically bonds with MIR4713HG. To the best of our knowledge, the function of miRNA in OTSCC remains unclear. To examine the impact of the expression level of let-7c-5p on OTSCC cells, the present study performed a series of rescue experiments. The results revealed that the addition of let-7c-5p counteracted the improved cell proliferation and clonogenicity induced by MIR4713HG, and suppressed the increased metastasis potential in OTSCC cells caused by MIR4713HG overexpression. Furthermore, it was revealed that expression of let-7c-5p was downregulated in OTSCC and negatively correlated with MIR4713HG. According to the results of the bioinformatic prediction, it was revealed that TMC7 directly bound with let-7c-5p. The expression pattern of TMC7 exhibited the opposite to that of let-7c-5p. The administration of let-7c-5p mimics or inhibitor decreased or increased the expression level of TMC7, respectively. Furthermore, the overexpression of MIR4713HG could increase the expression of TMC7, and in rescue experiments, the knockdown of TMC7 could counteract the effect induced by MIR4713HG overexpression on OTSCC cells.

In conclusion, the present study demonstrated that MIR4713HG was significantly upregulated in both OTSCC tissues and cell lines. MIR4713HG acted as a pro-tumor factor, facilitating cell proliferation and metastasis. Furthermore, the present study identified that MIR4713HG directly bound with let-7c-5p, and that let-7c-5p regulated malignant behaviors of OTSCC via affecting the expression of TMC7. Collectively, MIR4713HG aggravated the malignant behaviors in OTSCC via affecting the let-7c-5p/TMC7 signaling pathway, and could be a promising therapeutic target in OTSCC.

\section{Acknowledgements}

Not applicable.

\section{Funding}

The present study was financially supported by the Guangzhou Science and Technology Program Key Projects (grant no. 201802020018), the China Postdoctoral Science Foundation (grant no. 2019M652979), the Guangdong Science and Technology Program (grant no. 2019A1515010408), the Program of Stomatologic Hospital, Southern Medical University, China (grant no. PY2019003) and the Medical Scientific Research Foundation of Guangdong Province of China (grant no. B2017103).

\section{Availability of data and materials}

The datasets used and/or analyzed during the current study are available from the corresponding author on reasonable request.

\section{Authors' contributions}

$\mathrm{BJ}$ and $\mathrm{JZ}$ conceived the research idea and designed the whole experimental plan. BJ, XQ and SX performed the experiments. $\mathrm{BJ}$ and $\mathrm{XZ}$ analyzed the in vitro and in vivo results and discussed the findings with GC, who wrote the initial draft of the manuscript. XJ, JL and ZH collected and analyzed the data, revised and finalized the manuscript. GC and SX provided constructive suggestions for the work and proofread the language of the manuscript. All authors read and approved the final version of the manuscript.

\section{Ethics approval and consent to participate}

The research protocols associated with the experimental mice were approved by the Ethics Committee of the Southern Medical University. With approval from the institutional review board of the Joint Ethics Committee of the Southern Medical University Health Authority, freshly resected OTSCC tissues (20 pairs) and paraffin-embedded OTSCC sections were obtained from the archives of the Department of Oral Surgery, Stomatological Hospital, Southern Medical University (Guangzhou, China).

\section{Patient consent for publication}

Not applicable.

\section{Competing interests}

The authors declare that they have no competing interests.

\section{References}

1. Siegel RL, Miller KD and Jemal A: Cancer statistics, 2015. CA Cancer J Clin 65: 5-29, 2015.

2. Franceschi D, Gupta R, Spiro RH and Shah JP: Improved survival in the treatment of squamous carcinoma of the oral tongue. Am J Surg 166: 360-365, 1993.

3. Wong WM, Parvathaneni U, Jewell PD, Martins RG, Futran ND, Laramore GE and Liao JJ: Squamous cell carcinoma of the oral tongue in a patient with Fanconi anemia treated with radiotherapy and concurrent cetuximab: A case report and review of the literature. Head Neck 35: E292-E298, 2013.

4. Amichetti M: Squamous cell carcinoma of the oral tongue in patients less than fifteen years of age. Report of a case and review of the literature. J Craniomaxillofac Surg 17: 75-77, 1989.

5. Miller KD, Nogueira L, Mariotto AB, Rowland JH, Yabroff KR, Alfano CM, Jemal A, Kramer JL and Siegel RL: Cancer treatment and survivorship statistics, 2019. CA Cancer J Clin 69: 363-385, 2019. 
6. Fakih AR, Rao RS, Borges AM and Patel AR: Elective versus therapeutic neck dissection in early carcinoma of the oral tongue. Am J Surg 158: 309-313, 1989.

7. He Q, Chen Z, Dong Q, Zhang L, Chen D, Patel A, Koya A, Luan X, Cabay RJ, Dai Y, et al: MicroRNA-21 regulates prostaglandin E2 signaling pathway by targeting 15-hydroxyprostaglandin dehydrogenase in tongue squamous cell carcinoma. BMC Cancer 16: $685,2016$.

8. St Laurent G, Wahlestedt C and Kapranov P: The landscape of long noncoding RNA classification. Trends Genet 31: 239-251, 2015.

9. Chen LL: Linking long noncoding RNA localization and function. Trends Biochem Sci 41: 761-772, 2016.

10. Marchese FP, Raimondi I and Huarte M: The multidimensional mechanisms of long noncoding RNA function. Genome Biol 18 206, 2017.

11. Sun M, Nie FQ, Wang ZX and De W: Involvement of lncRNA dysregulation in gastric cancer. Histol Histopathol 31: 33-39, 2016

12. Xia P, Gu R, Zhang W and Sun YF: IncRNA CEBPA-AS1 overexpression inhibits proliferation and migration and stimulates apoptosis of OS cells via notch signaling. Mol Ther Nucleic Acids 19: 1470-1481, 2020

13. Cao Y, Xiong JB, Zhang GY, Liu Y, Jie ZG and Li ZR: Long noncoding RNA UCA1 regulates PRL-3 expression by sponging MicroRNA-495 to promote the progression of gastric cancer. Mol Ther Nucleic Acids 19: 853-864, 2020.

14. Liu SJ, Malatesta M, Lien BV, Saha P, Thombare SS, Hong SJ, Pedraza L, Koontz M, Seo K, Horlbeck MA, et al: CRISPRi-based radiation modifier screen identifies long non-coding RNA therapeutic targets in glioma. Genome Biol 21: 83, 2020.

15. Ren X, Chen C, Luo Y, Liu M, Li Y, Zheng S, Ye H, Fu Z, Li M, Li Z and Chen R: IncRNA-PLACT1 sustains activation of NF- $\kappa B$ pathway through a positive feedback loop with $\mathrm{I} \kappa \mathrm{B} \alpha / \mathrm{E} 2 \mathrm{~F} 1$ axis in pancreatic cancer. Mol Cancer 19: 35, 2020.

16. Pan J, Fang S, Tian H, Zhou C, Zhao X, Tian H, He J, Shen W, Meng X, Jin X and Gong Z: lncRNA JPX/miR-33a-5p/Twist 1 axis regulates tumorigenesis and metastasis of lung cancer by activating Wnt/ $\beta$-catenin signaling. Mol Cancer 19: 9, 2020.

17. Xiong L, Tang Y, Tang J, Liu Z and Wang X: Downregulation of lncRNA HOTTIP suppresses the proliferation, migration, and invasion of oral tongue squamous cell carcinoma by regulation of HMGA2-Mediated Wnt/ $\beta$-catenin pathway. Cancer Biother Radiopharm 35: 720-730, 2020.

18. Mu M, Li Y, Zhan Y, Li X and Zhang B: Knockdown of HOXA transcript at the distal tip suppresses the growth and invasion and induces apoptosis of oral tongue squamous carcinoma cells Onco Targets Ther 11: 8033-8044, 2018.

19. Robinson MD, McCarthy DJ and Smyth GK: edgeR: A bioconductor package for differential expression analysis of digital gene expression data. Bioinformatics 26: 139-140, 2010.

20. Tang X, Shi L, Xie N, Liu Z, Qian M, Meng F, Xu Q, Zhou M, Cao X, Zhu WG and Liu B: SIRT7 antagonizes TGF- $\beta$ signaling and inhibits breast cancer metastasis. Nat Commun 8: 318, 2017.

21. Wei C, Yang C, Wang S, Shi D, Zhang C, Lin X, Liu Q, Dou R and Xiong B: Crosstalk between cancer cells and tumor associated macrophages is required for mesenchymal circulating tumor cell-mediated colorectal cancer metastasis. Mol Cancer 18: 64, 2019.
22. Livak KJ and Schmittgen TD: Analysis of relative gene expression data using real-time quantitative PCR and the 2(-Delta Delta C(T)) method. Methods 25: 402-408, 2001

23. Wang S, Ke H, Zhang H, Ma Y, Ao L, Zou L, Yang Q, Zhu H, Nie J, Wu C and Jiao B: IncRNA MIR100HG promotes cell proliferation in triple-negative breast cancer through triplex formation with p27 loci. Cell Death Dis 9: 805, 2018.

24. Cui Y, Song Y, Yan S, Cao M, Huang J, Jia D, Liu Y, Zhang S, Fan W, Cai L, et al: CUEDC1 inhibits epithelial-mesenchymal transition via the T $\beta R I /$ Smad signaling pathway and suppresses tumor progression in non-small cell lung cancer. Aging (Albany NY) 12: 20047-20068, 2020.

25. Chen T, You Y, Jiang H and Wang ZZ: Epithelial-mesenchymal transition (EMT): A biological process in the development, stem cell differentiation, and tumorigenesis. J Cell Physiol 232: 3261-3272, 2017.

26. Paraskevopoulou MD, Vlachos IS and Hatzigeorgiou AG: DIANA-TarBase and DIANA suite tools: Studying experimentally supported microRNA targets. Curr Protoc Bioinformatics 55: 12.14.1-12.14.18, 2016.

27. Lewis BP, Burge CB and Bartel DP: Conserved seed pairing, often flanked by adenosines, indicates that thousands of human genes are microRNA targets. Cell 120: 15-20, 2005.

28. Bray F, Ferlay J, Soerjomataram I, Siegel RL, Torre LA and Jemal A: Global cancer statistics 2018: GLOBOCAN estimates of incidence and mortality worldwide for 36 cancers in 185 countries. CA Cancer J Clin 68: 394-424, 2018.

29. Camisasca DR, Silami MA, Honorato J, Dias FL, de Faria PA and Lourenco Sde Q: Oral squamous cell carcinoma: Clinicopathological features in patients with and without recurrence. ORL J Otorhinolaryngol Relat Spec 73: 170-176, 2011

30. Lindenblatt Rde C, Martinez GL, Silva LE, Faria PS, Camisasca DR and Lourenco Sde Q: Oral squamous cell carcinoma grading systems-analysis of the best survival predictor. J Oral Pathol Med 41: 34-39, 2012.

31. Chen YH, Chien CY, Fang FM, Huang TL, Su YY, Luo SD, Huang CC, Lin WC and Li SH: Nox4 overexpression as a poor prognostic factor in patients with oral tongue squamous cell carcinoma receiving surgical resection. J Clin Med 7: 497, 2018.

32. Wang W, Li J, Zhang Z, Ma H, Li Q, Yang H, Li M and Liu L: Genome-wide analysis of acute traumatic spinal cord injury-related RNA expression profiles and uncovering of a regulatory axis in spinal fibrotic scars. Cell Prolif 54: e12951, 2021.

33. Zhang M, Han Y, Zheng Y, Zhang Y, Zhao X, Gao Z and Liu X: ZEB1-activated LINC01123 accelerates the malignancy in lung adenocarcinoma through NOTCH signaling pathway. Cell Death Dis 11: $981,2020$.

34. Hong W, Ying H, Lin F, Ding R, Wang W and Zhang M: lncRNA LINC00460 silencing represses EMT in colon cancer through downregulation of ANXA2 via upregulating miR-433-3p. Mol Ther Nucleic Acids 19: 1209-1218, 2020.

This work is licensed under a Creative Commons Attribution-NonCommercial-NoDerivatives 4.0 International (CC BY-NC-ND 4.0) License. 